\title{
Aberrant Tendency of Noncurrent Emotional Experiences in Individuals at Ultra-High Risk for Psychosis
}

\author{
Eunchong Seo ${ }^{1,2}$, Minji Bang ${ }^{2,3} \bowtie$, Eun Lee ${ }^{1,2}$, and Suk Kyoon $\mathrm{An}^{1,2,4} \bowtie$ \\ ${ }^{1}$ Department of Psychiatry, Yonsei University College of Medicine, Severance Hospital, Seoul, Republic of Korea \\ ${ }^{2}$ Section of Self, Affect, and Neuroscience, Institute of Behavioral Science in Medicine, Yonsei University College of Medicine, Seoul, Republic of Korea \\ ${ }^{3}$ Department of Psychiatry, CHA Bundang Medical Center, CHA University, Seongnam, Republic of Korea \\ ${ }^{4}$ Graduate Program in Cognitive Science, Yonsei University, Seoul, Republic of Korea
}

Objective This study aimed to investigate whether aberrant tendency of noncurrent emotion was present in individuals at ultra-high risk (UHR) for psychosis and to explore its associations with various clinical profiles.

Methods Fifty-seven individuals at UHR and 49 normal controls were enrolled. The tendency of experiencing noncurrent emotion was assessed using various noncurrent emotional self-reported formats, including trait [Neuroticism and Extraversion of the Eysenck Personality Questionnaire], hypothetical (Chapman's Revised Physical and Social Anhedonia Scales), and retrospective [AnhedoniaAsociality Subscale of the Scale for the Assessment of Negative Symptoms (SANS)] measures. Self-related beliefs (Self-Perception Scale), clinical positive and negative symptoms (SA Positive Symptoms and SANS), psychosocial function (Global Functioning Scale: Role Function and Global Functioning Scale: Social Function) were also examined.

Results Subjects at UHR for psychosis reported more trait unpleasant and less trait pleasant emotions, more hypothetical physical and social anhedonia, and more retrospective anhedonia than normal controls. In UHR, self-perception was correlated to trait unpleasant emotion and hypothetical physical and social anhedonia. Negative symptoms in UHR were associated with hypothetical physical anhedonia and retrospective anhedonia. Global social functioning was related to trait pleasant emotion, hypothetical physical and social anhedonia, and retrospective anhedonia. Neurocognitive function, positive symptoms, and global role functioning were not related with any noncurrent emotional experience measures in UHR.

Conclusion These findings suggest that the aberrant tendency of noncurrent emotional experience may be present at the 'putative' prodromal phase and are grossly associated with self-related beliefs and psychosocial functioning but not neurocognitive functioning.

Psychiatry Investig 2018;15(9):876-883

Key Words Emotional experience, Noncurrent emotion, Self-perception, Neurocognition, Psychosocial function, Ultra-high risk for psychosis.

\section{INTRODUCTION}

Disturbance of personality and emotional characteristics, one of the key clinical features of patients with schizophrenia ${ }^{1,2}$ is manifested in various ways including a diminished capacity to experience pleasure (i.e., anhedonia) and height-

Received: February 22, 2018 Revised: July 12, 2018

Accepted: July 29, 2018

$\triangle$ Correspondence: Suk Kyoon An, MD, PhD

Department of Psychiatry, Yonsei University College of Medicine, 50-1 Yonsei-ro, Seodaemun-gu, Seoul 03722, Republic of Korea

Tel: +82-2-2228-1585, Fax: +82-2-313-0891, E-mail: ansk@yuhs.ac

$\triangle$ Correspondence: Minji Bang, MD, PhD

Department of Psychiatry, CHA Bundang Medical Center, CHA University, 59 Yatap-ro, Bundang-gu, Seongnam 13496, Republic of Korea Tel: +82-31-780-5865, Fax: +82-31-780-5862, E-mail: minji.bang@gmail.com

(a) This is an Open Access article distributed under the terms of the Creative Commons Attribution Non-Commercial License (https://creativecommons.org/licenses/bync/4.0) which permits unrestricted non-commercial use, distribution, and reproduction in any medium, provided the original work is properly cited. ened sensitivity to negative emotional experiences. ${ }^{3-5}$ Such aberrations have been suggested to be associated with worsening of clinical symptoms and functioning of patients with schizophrenia, thereby, causing chronic distress from emotional experiences and isolation from social activities and interpersonal relationships. ${ }^{6,7}$ Furthermore, some recent studies have shown that emotional dispositions affect not only the clinical manifestation and illness course during the prodromal phase but also the onset of fully developed psychosis. ${ }^{4,89}$ Therefore, investigating the emotional dispositions and related psychological factors in individuals at ultra-high risk (UHR) for psychosis could help prevent the development of schizophrenia spectrum psychosis and improve clinical status.

A tendency to experience less pleasant but more unpleasant emotion is assessed by various measures, which could be 
conceptually divided by whether they mainly evaluate current or noncurrent emotional experiences. ${ }^{5,10}$ Widely used measurements, including self-reported questionnaires and interview-based assessments, usually focus on noncurrent emotion, rather than current feelings experienced in laboratorybased, controlled environments. However, the measures of noncurrent emotion also include components on emotional experiences encompassing past emotions, expectation regarding future emotion, or inferences on emotions in specific situations. The accessibility model of emotional self-report, which provides a cognitive framework to conceptualize noncurrent emotion, ${ }^{10,11}$ suggests that people access at least four types of knowledge when they report noncurrent feelings: experiential knowledge, episodic memory, situation-specific belief, and identity-related belief. According to this model, it would be important to take into account related psychological components such as neurocognitive function, personality trait, and self-related belief in order to elucidate the characteristics of noncurrent emotional experiences in individuals at UHR for psychosis.

Noncurrent pleasant emotion has been investigated using the measures of trait affectivity and hypothetical and retrospective anhedonia. ${ }^{5,10,12}$ For trait affectivity, self-reports on some personality traits [e.g., Extraversion of the Eysenck Personality Questionnaire (EPQ)] have been used to assess noncurrent emotional tendency (pleasant trait affectivity). Hypothetical anhedonia can be measured by the self-report of one's emotional experiences toward specific physical and social situations. On the other hand, retrospective anhedonia is assessed based on an interviewer's appraisal of an interviewee's reports of recent reactions to emotional experiences. These measures evaluate not only the capacity to experience pleasure but also self-related knowledge, one's expectations of emotional experience in specific situations, and the ability to recall past events regarding emotional experiences. Therefore, the measured noncurrent pleasant emotion would be associated with one's identity-related beliefs, situation-specific beliefs, and neurocognitive function., ${ }^{5,10,12}$ Given that patients with schizophrenia have been found to show a relatively preserved capacity to feel current pleasant emotion compared to healthy individuals, ${ }^{10,12}$ distorted self-related belief and deficits in neurocognitive function would play a more critical role in schizophrenia-related anhedonia. Meanwhile, there have only been few studies on unpleasant emotional experiences, since they have not been labeled as anhedonia. One of the typical noncurrent measures for unpleasant emotional tendency is self-reports on trait affectivity (e.g., Neuroticism of EPQ). Some previous studies on unpleasant trait affectivity have shown that unpleasant emotional tendency in schizophrenia exposed the patients to chronic distress ${ }^{5}$ and wors- ened clinical symptoms. ${ }^{13-15}$

Although there have been numerous previous studies on the association between the aberrant tendency of emotion and clinical symptoms, the findings have not been consistent and appear to depend on the characteristics of each measurement. Some studies have shown significant relationships between unpleasant trait affectivity and positive symptoms ${ }^{13-15}$ and between pleasant trait affectivity and negative symptoms, ${ }^{16}$ but this has not always been the case. ${ }^{5}$ Studies using questionnaires for hypothetical anhedonia (e.g., Chapman's Revised Physical and Social Anhedonia Scales) have found no correlation with positive symptoms ${ }^{17-19}$ and inconsistent results regarding negative symptoms. ${ }^{18,20}$ Other prior studies on the association between personality trait affectivity and psychosocial functioning have shown that higher unpleasant trait affectivity correlated with decreased quality of life $\mathrm{e}^{16,21,22}$ and poor occupational functioning. ${ }^{21,23}$ In addition, higher pleasant trait affectivity was found to be associated with increased quality of life and better social functioning. ${ }^{21,24}$ For hypothetical anhedonia, it has been consistently reported that more severe anhedonia is correlated with worse community functioning. ${ }^{19,25-27}$ Meanwhile, there have been only few studies on the aberrant tendency of emotional experiences in individuals at UHR for psychosis, although increased severity of anhedonia in this population has been previously reported. ${ }^{28-30}$ It has not been sufficiently explored whether the aberrant tendency of noncurrent emotional experience during the prodromal phase is significantly associated with the underlying factors including neurocognitive function, skewed self-related belief, clinical symptoms, and psychosocial functioning.

In this study, we aimed to investigate whether the aberrant tendency of noncurrent emotional experience presented during the prodromal phase using comprehensive measures of trait affectivity, and hypothetical and retrospective emotional experience. Individuals at UHR for psychosis were expected to show less pleasant emotion and more unpleasant emotion. Moreover, we investigated the association of this aberrant tendency with psychosocial factors including self-related beliefs, neurocognitive function, the severity of positive and negative symptoms, and psychosocial function, thereby attempting to construct a comprehensive picture of multiple factors related to the aberrant tendency of noncurrent emotional experience.

\section{METHODS}

\section{Subjects}

Fifty-seven (male 35, female 22) individuals at UHR and 49 (male 25, female 24) normal controls (NC) were recruited. individuals at UHR for psychosis were included based on the Criteria of Prodromal Symptoms from the Structured Inter- 
view for Prodromal Syndromes. ${ }^{31}$ For normal controls, participants with past or current diagnosis for any Axis I disorder, past or current drug use disorder, and neurological disorders were excluded. All participants were assessed for psychiatric disorders using the Structured Clinical Interview for DSMIV. ${ }^{32,33}$ Written informed consent was obtained from all participants after procedures had been fully explained. Additionally, for participants under the age of 18 years, we obtained informed consent from their parents. The study was reviewed and approved by the Institutional Review Board of Severance Hospital (IRB No. 4-2014-0744, 4-2016-0570).

\section{Measures}

\section{Assessment of the noncurrent emotional experience}

We used the Neuroticism and Extraversion subscales of the EPQ, Chapman's Revised Physical and Social Anhedonia Scales, and the Anhedonia-Asociality subscale of the Scale for the Assessment of Negative Symptoms (SANS). ${ }^{34}$ The characteristics of these measurements are summarized in Table 1 according to the accessibility model of emotional selfreport. ${ }^{10,11}$

The EPQ, a self-reported personality measurement, consists of four subscales (Psychoticism, Extraversion, Neuroticism, and Lie) with a total of 81 items that assess personality traits. We used the Neuroticism and Extraversion subscales, which are known to represent trait affectivity. The Neuroticism subscale consists of 26 items that assess emotional instability characterized by propensity for a high level of negative affect such as depression and anxiety. The Extraversion subscale is composed of 18 items that assess such characteristics as being outgoing, talkative, and having a high level of positive affect. We used Korean version of EPQ, ${ }^{35}$ and it was known to show good reliability and validity. The internal consistency in the present study was as follows: Extraversion, 0.89; Neuroticism, 0.93 .

The self-reported Chapman's Revised Physical and Social Anhedonia Scales were used to measure anhedonia on the hypothetical emotional experiences. The Revised Physical An- hedonia scale is composed of 61 items that assess deficits in the ability to experience pleasure from typically pleasurable physical stimuli such as food, sex, and scenes. The Revised Social Anhedonia scale consists of 40 items that assess deficits in the ability to experience pleasure from nonphysical stimuli such as talking and exchanging feelings. We used Korean version of Chapman's Revised Physical and Social Anhedona Scales which is widely used in Korean psychiatric research. ${ }^{36-38}$ The internal consistency in the present study was as follows: Revised Physical Anhedonia, 0.92; Revised Social Anhedonia, 0.93. The Anhedonia-Asociality subscale of the SANS was used for the retrospective assessment of anhedonia on emotional experiences. It is an interview-based measure, and the interviewer assesses the interviewee's deficits on "Recreational interests and activities," "Sexual interest and activities," "Ability to feel intimacy and closeness," and "Relationships with friends and Peers" in past month.

\section{Self-Perception Scale}

The Self-Perception Scale ${ }^{39}$ is a self-report measure assessing the belief pattern concerning how individuals perceive themselves. The Self-Perception Scale is composed of 30 adjectives regarding personality traits, which are selected from the results (400 adjectives) of the "analysis of Korean personality trait adjectives." ${ }^{40}$ Twenty-five adjectives concern positive traits (for example, "polite" and "kind") and five concern negative ones (for example, "arrogant" and "selfish"). Each item is scored from 0 (not at all) to 4 (definitely yes). As the negative trait items were reversely scored, the total score reflected positive self-perception. The Self-Perception Scale has good validity and reliability attributes and is widely used in Korean psychiatric and psychological research. The scale's internal consistency in this study was 0.88 .

\section{Neurocognitive assessment}

For the assessment of neurocognitive function, we used a comprehensive neurocognitive battery, as described in our previous study. ${ }^{41}$ The battery consists of the California Verbal Learning test, 3-7 continuous Performance Test, Verbal and

Table 1. Characteristics of the scales of the noncurrent emotional experience

\begin{tabular}{llll}
\hline & \multicolumn{1}{c}{$\begin{array}{c}\text { Neuroticism subscale } \\
\text { of EPQ }\end{array}$} & $\begin{array}{c}\text { Extraversion subscale } \\
\text { of EPQ }\end{array}$ & $\begin{array}{c}\text { Chapman's revised physical and } \\
\text { social anhedonia scale }\end{array}$ \\
\hline Object & Affective trait & Affective trait & Hypothetical anhedonia \\
Emotional valence & Unpleasant emotion & Pleasant emotion & Pleasant emotion \\
Method & Self-report measures & Self-report measures & Self-report measures \\
Based knowledge & Semantic knowledge & Semantic knowledge & Semantic knowledge \\
Information source & Identity-related belief & Identity-related belief & Situation-related belief \\
& & & Identity-related belief
\end{tabular}

EPQ: Eysenck Personality Questionaire, SANS: Scale for Negative Symptom scale 
Visuospatial 2-Back Test, Rey Complex Figure Test, Controlled Word Association Test, Figure Fluency Test, Trail Making Test (parts A and B), Wisconsin Card Sorting Test, and the Stroop Test. Each neurocognitive test score was transformed into the equivalent $\mathrm{z}$ score to allow for comparisons across the different neurocognitive tests. All tests were categorized into five neurocognitive domains (verbal memory, attention and working memory, spatial memory, psychomotor speed, and executive function), which were determined based on the confirmation of factor structure. ${ }^{41}$ In the present study, the internal consistency of each domain was as follows: verbal memory, 0.89 ; attention and working memory, 0.77 ; spatial memory, 0.97 ; psychomotor speed, 0.62 ; and executive function, 0.60 .

Assessment of psychopathology and social and role function

The psychopathology and severity of symptoms in UHR were assessed using the Scale for the Assessment of Positive Symptoms (SAPS) ${ }^{42}$ and the SANS. ${ }^{34}$

To assess psychosocial function, we used Global functioning: Social and Global functioning: Role. ${ }^{43}$ The scores of both scales range from 1 (extreme dysfunction) to 10 (superior function). Both scales include focused and detailed anchor points for each rating interval to increase reliability. Each scale is includes three scores: Current functioning (the lowest level of functioning in the past month) and the Lowest and Highest level of functioning over the past year. In this study, we only assessed Current functioning. Global Functioning: Social Scale assesses the quality and quantity of social relationships. The interviewer inquires after the subject's relationship with friends, family, and lovers. Interactions with people other than family members receive higher scores than interactions limited to family members. The Global Functioning: Role Scale pertains to performance in school, work, or at home. When subjects are capable of functioning independently in these roles, they receive a high score in the Global Functioning: Social scale. In our prior study, ${ }^{44}$ the Global functioning: Social and Global functioning: Role were plausible in Korean individuals at UHR for psychosis.

\section{Statistical analysis}

To compare the scale scores on noncurrent emotional experience (EPQ, Chapman's Anhedonia Scale, and Anhedonia-Asociality Subscale of SANS) between the NC and UHR groups, independent-sample $t$ tests were used. Pearson correlations of scales about emotional experience (EPQ, Chapman's Anhedonia Scale, Anhedonia-Asociality Subscale of SANS) with self-perception scale, five domains of neurocognitive function, psychopathology (SAPS, SANS), and Global functioning (social, role) were conducted in UHR group. We used Holm-Bonferroni correction ${ }^{45}$ to revise p-value for Pearson correlation analysis. A significance level of $p$ less than 0.05 was used for all test.

\section{RESULTS}

\section{Demographic and clinical profiles of participants}

There was no difference between the two groups in demographics (Table 2). The UHR group's Self-Perception Scale score was lower than that of the NC group $(\mathrm{p}<0.001)$. The UHR group showed significantly lower neurocognitive function than the NC group in the verbal memory domain $(\mathrm{p}<$ $0.001)$, attention and working memory domain $(\mathrm{p}=0.02)$, and psychomotor speed domain ( $\mathrm{p}=0.004)$. The UHR group's social and role functions were significantly worse than those of the NC group $(\mathrm{p}<0.001)$.

Table 2. The demographics and clinical profiles of subjects

\begin{tabular}{|c|c|c|c|}
\hline & $\begin{array}{c}\mathrm{NC} \\
(\mathrm{N}=43)\end{array}$ & $\begin{array}{c}\text { UHR } \\
(\mathrm{N}=57)\end{array}$ & $\mathrm{p}$ \\
\hline Age (years) & $21.5(3.6)$ & $20.5(3.5)$ & 0.17 \\
\hline $\operatorname{Sex}(M / F)$ & $25 / 18$ & $35 / 22$ & \\
\hline Education (years) & $13.5(1.9)$ & $13.1(1.9)$ & 0.32 \\
\hline \multicolumn{4}{|l|}{ SIPS criteria } \\
\hline APSS & & 39 & \\
\hline BIPS & & 1 & \\
\hline APSS+BIPS & & 5 & \\
\hline APSS+GRDS & & 12 & \\
\hline Self-perception scale & $75.4(14.7)$ & $52.8(15.2)$ & $<0.001$ \\
\hline \multicolumn{4}{|l|}{ Neurocognitive function } \\
\hline Verbal memory & $0.2(0.7)$ & $-0.5(1.2)$ & $<0.001$ \\
\hline Attention-working memory & $0.1(0.6)$ & $-0.3(1.0)$ & 0.01 \\
\hline Spatial memory & $-0.1(1.0)$ & $-0.3(1.0)$ & 0.18 \\
\hline Psychomotor speed & $0.0(0.7)$ & $-0.4(0.7)$ & 0.003 \\
\hline Executive function & $0.1(0.5)$ & $-0.2(0.8)$ & 0.05 \\
\hline Positive symptoms of SAPS & $0.0(0.0)$ & $3.3(2.4)$ & $<0.001$ \\
\hline Negative symptoms of SANS & $0.4(0.9)$ & $8.4(4.1)$ & $<0.001$ \\
\hline Global functioning: social* & $8.5(0.8)$ & $4.6(1.5)$ & $<0.001$ \\
\hline Global functioning: role* & $8.1(0.8)$ & $5.3(1.7)$ & $<0.001$ \\
\hline \multicolumn{4}{|l|}{ Antipsychotic medications } \\
\hline Naïve/medicated & & $38 / 19$ & \\
\hline $\begin{array}{l}\text { Clorpromazine equivalent } \\
\text { dose }(\mathrm{mg} / \mathrm{d})^{\dagger}\end{array}$ & & $109.8(72.0)$ & \\
\hline
\end{tabular}

*some date (4 UHR) were missing, ${ }^{\dagger}$ Kroken et al. ${ }^{46}$

NC: normal control, UHR: ultra-high risk for psychosis, SIPS: Structured Interview for Prodromal Syndromes, APSS: Attenuated Positive Symptom Syndrome, BIPS: Brief Intermittent Psychotic Syndrome, GRDS: Genetic Risk and Deterioration Syndrome, SAPS: Scale for the Assessment of Positive Symptoms, SANS, Scale for the Assessment of Negative Symptoms 


\section{Comparison of emotional experience reports}

The EPQ (neuroticism, extraversion) and Anhedonia scales [Chapman Anhedonia Scale (Social, Physical)], AnhedoniaAsociality Subscale of the SANS for the NC and UHR groups are shown in Table 3. For unpleasant emotional experience, the UHR group had higher scores in the EPQ Neuroticism scale than the NC group ( $t=-7.5, \mathrm{DF}=97.1, \mathrm{p}<0.001)$. For pleasant emotional experience, individuals at UHR for psychosis had lower scores on the EPQ Extraversion scale ( $\mathrm{t}=6.34$, $\mathrm{DF}=97.9, \mathrm{p}<0.001)$, and higher score on Chapman's Physical $(\mathrm{t}=-5.0, \mathrm{DF}=98, \mathrm{p}<0.001)$ and Social Anhedonia Scale $(\mathrm{t}=$ $-8.9, \mathrm{DF}=98, \mathrm{p}<0.001)$, Anhedonia-Asociality Subscale of the SANS $(t=-11.6, D F=98, p<0.001)$ than the $N C$ group.

\section{Correlation analyses of affectivity with self-perception,} neurocognitive function, symptoms, and global functioning in ultra-high risk group (Table 4)

The Self-Perception Scale scores of the UHR group were significantly correlated with the Neuroticism scale of the of $\mathrm{EPQ}$, Chapman's revised physical and social anhedonia (all corrected $\mathrm{ps}<0.001)$. All neurocognitive function domains (verbal memory, attention and working memory, spatial memory, psychomotor speed, and executive function) were not correlated with any emotional experience scale score. Negative symptoms (SANS) were significantly correlated with Chapman's Revised Physical Anhedonia Scale (corrected $\mathrm{p}=0.04$ ) and the Anhedonia-Asociality subscale of the SANS (corrected $\mathrm{p}<0.001$ ). Global social function was significantly correlated with the Extraversion subscale of the EPQ (corrected $\mathrm{p}=0.04$ ), Chapman's Revised Physical Anhedonia (corrected $\mathrm{p}=0.001$ ), Chapman's Revised Social Anhedonia (corrected $\mathrm{p}=0.04$ ), and the Anhedonia-Asociality subscale of the SANS (corrected $\mathrm{p}<0.001$ ).

\section{DISCUSSION}

The present study revealed the aberrant tendency of noncurrent emotional experience using various measures of trait affectivity, hypothetical anhedonia, and retrospective anhedonia in individuals at UHR for psychosis. More importantly, this tendency was found to be grossly associated with selfrelated beliefs and psychosocial functioning but not with

Table 3. The comparisons of the noncurrent emotional experiences between UHR and NCs

\begin{tabular}{llrrrrr}
\hline & \multicolumn{1}{c}{ Scales } & NC (N=43) & UHR (N=57) & t & p & ES (Cohen's d) \\
\hline Unpleasant emotional experience & Neuroticism of EPQ & $7.6(5.0)$ & $16.8(7.3)$ & -7.5 & $<0.001$ & 1.45 \\
Pleasant emotional experience & Extraversion of EPQ & $11.8(4.0)$ & $5.5(5.5)$ & 6.6 & $<0.001$ & -1.30 \\
& Chapman's physical anhedonia scale & $13.9(7.6)$ & $24.2(11.9)$ & -5.0 & $<0.001$ & 1.01 \\
& Chapman's social anhedonia scale & $9.2(5.2)$ & $22.5(8.7)$ & -9.5 & $<0.001$ & 1.81 \\
& Anhedonia-asociality subscale of SANS & $0.1(0.4)$ & $2.6(1.4)$ & -13.1 & $<0.001$ & 2.32 \\
\hline
\end{tabular}

NC: normal control, UHR: ultra-high risk for psychosis, EPQ: Eysenck Personality Questionaire, SANS: Scale for Negative Symptom scale

Table 4. The correlations of noncurrent emotional experience with psychosocial factors in ultra-high risk for psychosis $(\mathrm{N}=57)$

\begin{tabular}{|c|c|c|c|c|c|}
\hline & \multirow{2}{*}{\multicolumn{2}{|c|}{$\begin{array}{c}\text { Unpleasant emotional experience } \\
\text { Trait affectivity }\end{array}$}} & \multicolumn{3}{|c|}{ Pleasant emotional experience } \\
\hline & & & \multicolumn{2}{|c|}{ Hypothetical anhedonia } & \multirow{2}{*}{$\begin{array}{c}\text { Retrospective } \\
\text { anhedonia } \\
\text { Anhedonia- } \\
\text { asociality(SANS) }\end{array}$} \\
\hline & $\begin{array}{c}\text { Neuroticism } \\
\text { of EPQ }\end{array}$ & $\begin{array}{c}\text { Extraversion } \\
\text { of EPQ }\end{array}$ & $\begin{array}{c}\text { Chapman's revised } \\
\text { physical anheonia scale }\end{array}$ & $\begin{array}{c}\text { Chapman's revised } \\
\text { social anhedonia scale }\end{array}$ & \\
\hline Self-perception scale & $-0.476^{\dagger}$ & 0.260 & $-0.520^{\dagger}$ & $-0.591^{\dagger}$ & -0.298 \\
\hline Verbal memory & 0.120 & -0.073 & 0.155 & 0.286 & -0.076 \\
\hline Attention-working memory & 0.200 & -0.031 & 0.139 & 0.046 & -0.272 \\
\hline Spatial memory & 0.287 & 0.045 & 0.207 & 0.179 & 0.023 \\
\hline Psychomotor speed & -0.08 & 0.222 & -0.097 & -0.216 & -0.036 \\
\hline Executive function & -0.085 & -0.109 & 0.116 & 0.097 & -0.133 \\
\hline SAPS & 0.148 & -0.075 & 0.333 & 0.069 & 0.380 \\
\hline SANS & 0.156 & -0.201 & $0.426^{*}$ & 0.358 & $0.862^{\dagger}$ \\
\hline Global function_role & 0.010 & 0.199 & -0.258 & -0.104 & -0.398 \\
\hline Global function_social & -0.173 & $0.442^{*}$ & $-0.559^{\dagger}$ & $-0.438^{*}$ & $-0.596^{\dagger}$ \\
\hline
\end{tabular}

Global function_role, Global function_social: 4 missing data. ${ }^{*}$ Holm-Bonferroni corrected $\mathrm{p}<0.05$, ${ }^{\dagger}$ Holm-Bonferroni corrected $\mathrm{p}<0.01$. EPQ: Eysenck Personality Questionaire, SANS: Scale for Negative Symptom scale 
neurocognitive functioning in individuals at UHR for psychosis.

Noncurrent emotional tendency, which is to experience less pleasant emotion and more unpleasant emotion, was shown in individuals at UHR for psychosis using comprehensive psychological measures. This aberrant tendency was generally consistent with the results of prior studies involving patients with schizophrenia ${ }^{3,4}$ and individuals at UHR for psychosis. ${ }^{9,30}$ In particular, aberrant emotional tendency was found across three dimensions of noncurrent emotional experiences including self-reported trait affectivity, subjective hypothetical emotional disposition, and interview-based objective appraisals on retrospective emotional events. To integrate our findings of UHR for psychosis and prior huge study's findings of noncurrent emotional experiences in patients with recent-onset schizophrenia, ${ }^{47,48}$ it may suggest that the noncurrent emotional experience is already aberrant from the 'putative' prodromal phase of schizophrenia-spectrum psychosis and continued after onset of schizophrenia. Therefore, proper intervening strategies for the aberrant tendency of noncurrent emotion would be necessary to be started before the onset of overt psychotic symptoms to help prevent the aggravation of symptoms and fully developed psychosis. ${ }^{6-9}$

Self-related beliefs, which were assessed with the Self-Perception Scale, were found to be associated with unpleasant trait affectivity related to neuroticism, but not with pleasant trait affectivity related to extraversion. The inverse association between neuroticism and positive self-perception found in this study corresponds to the accessibility model of emotional self-reports. ${ }^{10,11}$ On the other hand and contrary to our expectations, extraversion was not found to be associated with positive self-perception. This may be because the items measuring extraversion in the EPQ consist of questions that are not directly related to pleasant emotion; for example, "Do you have many friends?" and "Are you talkative?"5 For hypothetical anhedonia, the severity of anhedonia measured using Chapman's Revised Physical and Social Anhedonia Scales was significantly associated with poor self-perception. This result is consistent with previous findings in patients with schizophrenia ${ }^{11}$ and implies that hypothetical anhedonia is affected by self-perception starting at the prodromal phase. This association may be explained on the basis of the accessibility model of emotional self-reports; hypothetical anhedonia is reportedly largely based on semantic knowledge, which has the advantage of utilizing situation-specific or self-related beliefs to respond to questionnaire items. ${ }^{10,11}$ Meanwhile, the Anhedonia-Asociality Subscale of the SANS did not show any significant association with self-related beliefs. The selfrelated beliefs of the subjects may not affect anhedonia-asociality since the time window that is covered by the SANS (during the past month) is not long enough to reflect self-related beliefs.

For neurocognitive function, all domains were not associated with pleasant and unpleasant emotional experience. This result seems to be contrary to prior findings suggesting that neurocognitive function, especially verbal memory and executive function, underlies the aberrant tendency of self-reported noncurrent emotion in patients with schizophrenia., ${ }^{5,10,12}$ Given that individuals at UHR for psychosis have been known to show intermediate neurocognitive function, which is not deteriorated as seen in schizophrenia, ${ }^{47}$ the neurocognitive capacity of individuals at UHR for psychosis may not exert much influence on noncurrent emotional experience. Alternatively, noncurrent emotional experience may be independently manifested with a decline of neurocognitive function from the putative prodromal phase of schizophrenia. As previous findings using self-reports ${ }^{47,49}$ and psychophysiological measures of laboratory-based emotional experiences ${ }^{49}$ were shown to be diminished in individuals at UHR for psychosis, unlike established schizophrenia, the aberrant tendency of noncurrent emotion would be linked to current emotional experiences during the prodromal phase.

Regarding the clinical symptoms, positive symptoms were not correlated with noncurrent emotional tendency unlike negative symptoms. Our finding that positive symptoms were not associated with noncurrent emotional tendency is consistent with the results of prior studies involving patients with schizophrenia. ${ }^{5,13-15}$ The severity of negative symptoms was correlated with that of anhedonia, measured using Chapman's Revised Physical Anhedonia Scale and the AnhedoniaAsociality Subscale of the SANS, but not with trait affectivity. Since the SANS mainly evaluates the severity of symptoms during the past month, it may not be associated with trait affectivity that is formed based on lifelong experiences. Against our expectation, hypothetical anhedonia in social situations was not correlated with the severity of negative symptoms. This finding may be due to a discrepancy between subjective feelings and objective appraisals in social situations, which require more complex information processing than relatively simple physical conditions.

For psychosocial functioning, social function was generally associated with a decreased tendency of noncurrent pleasant emotion, while role function was not. Since most noticeable emotions are prominently experienced during social interactions, it is not surprising that social function was found to be associated with aberrant noncurrent emotional tendency in individuals at UHR for psychosis. In particular, social function showed a correlation only with pleasant emotional experience in our individuals at UHR for psychosis, suggesting that impaired social interactions in individuals at UHR for 
psychosis may be associated with a diminished capacity to anticipate pleasure on the basis of aberrant noncurrent emotional experience. The finding of no association with role function in individuals at UHR for psychosis was not consistent with the results of prior studies, which showed a correlation between unpleasant trait affectivity and occupational functioning in patients with schizophrenia. ${ }^{23}$

\section{Limitations}

First, we did not assess current emotional experience. However, we used comprehensive measures that reflect various aspects of noncurrent emotional experiences. Second, since our study was cross-sectional, we were not able to elucidate the causality between the emotional tendency and related psychosocial factors. In the future, follow-up studies will be needed to identify the causality between emotional tendencies and self-related beliefs, social function, and negative symptoms. Third, unpleasant emotional experience was not assessed to the same degree with pleasant emotional experience, as fewer instruments pertained to its measurement. Forth, we did not assess depressive and anxiety symptoms simultaneously. According to some recent studies, ${ }^{49-51}$ depressive and anxiety symptoms may affect emotional experience of individuals at UHR for psychosis. To the best of our knowledge, however, this is the first study to examine noncurrent emotional experience in individuals at UHR for psychosis concurrently using comprehensive measures of trait affectivity, hypothetical anhedonia, and retrospective anhedonia. Furthermore, we investigated the possible associations of aberrant tendency of noncurrent emotion with psychosocial factors of self-related beliefs, multiple neurocognitive functional domains, psychopathology, and psychosocial functioning.

In summary, we found that the aberrant tendency of noncurrent emotion was present at the UHR phase prior to the onset of fully developed schizophrenia-spectrum psychosis. In addition, aberrant tendency of noncurrent emotion was found to be associated with poor self-perception, negative symptoms, and social function in individuals at UHR for psychosis. Neurocognitive function was not correlated with this aberrant tendency, unlike in schizophrenia. Our findings may suggest that psychosocial intervention for individuals at UHR for psychosis should be different from that targeting patients with schizophrenia, which mainly focuses on impaired neurocognitive function. To develop more proper intervening strategies for individuals at UHR for psychosis, more studies on the aberrant tendency of noncurrent emotion and related factors will be needed.

\section{Acknowledgments}

This work was supported by the Basic Science Research Program through the National Research Foundation of Korea (NRF) funded by the Ministry of Science, ICT \& Future Planning, Republic of Korea (Grant number: 2017R1A2B3008214).

\section{REFERENCES}

1. Kraepelin E. Dementia Praecox and Paraphrenia. Huntington, NY: Robert E Krieger Publishing Company; 1971.

2. Bleuler E. Dementia Praecox or the Group of Schizophrenias. New York: International University Press; 1911/1950.

3. Simonsen E. Personality and Psychosis. In: Johannessen JO, Martindale BV, Cullberg J, Editors. Evolving Psychosis. East Sussex, UK: Routledge, 2006, p.35-48.

4. Smith TE, Shea MT, Schooler NR, Levin H, Deutsch A, Grabstein E. Personality traits in schizophrenia. Psychiatry 1995;58:99-112.

5. Horan WP, Blanchard JJ, Clark LA, Green MF. Affective traits in schizophrenia and schizotypy. Schizophr Bull 2008;34;856-874.

6. Krause R, Steimer-Krause E, Hufnagel H. Expression and experience of affects in paranoid schizophrenia. Eur Rev Appl Psychol 1992; 42:131-140.

7. Hooley JM, Richters JE, Weintraub S, Neale JM. Psychopathology and marital distress: the positive side of positive symptoms. J Abnorm Psychol 1987;96:27-33.

8. Torrey FT, Bowler AW, Taylor EH. Schizophrenia and Manic Depressive Disorder: The Biological Roots of Mental Illness. New York, NY: Basic Books; 1994.

9. Hulbert CA, Jackson HJ, McGorry PD. Relationship between personality and course and outcome in early psychosis: A review of the literature. Clin Psychol Rev1996;8:707-727.

10. Strauss GP, Gold JM. A new perspective on anhedonia in schizophrenia. Am J Psychiatry 2012;169:364-373.

11. Robinson MD, Clore GL. Belief and feeling: evidence for an accessibility model of emotional self-report. Psychol Bull 2002;128:934-960.

12. Horan WP, Kring AM, Blanchard JJ. Anhedonia in schizophrenia: a review of assessment strategies. Schizophr Bull 2006;32:259-273.

13. Lysaker PH, Lancaster RS, Nees MA, Davis LW. Neuroticism and visual memory impairments as predictors of the severity of delusions in schizophrenia. Psychiatry Res 2003;119:287-292.

14. Lysaker PH, Wilt MA, Plascak-Hallberg CD, Brenner CA, Clements CA. Personality dimensions in schizophrenia: associations with symptoms and coping. J Nerv Ment Dis 2003;191:80-86.

15. Lysaker PH, Bell MD, Kaplan E, Greig TC, Bryson GJ. Personality and psychopathology in schizophrenia: the association between personality traits and symptoms. Psychiatry 1999;62:36-48.

16. Herran A, Sierra-Biddle D, Cuesta MJ, Sandoya M, Vazquez-Barquero JL. Can personality traits help us explain disability in chronic schizophrenia? Psychiatry Clin Neurosci 2006;60:538-545.

17. Blanchard JJ, HoranWP, Brown SA. Diagnostic differences in social anhedonia: a longitudinal study of schizophrenia and major depressive disorder. J Abnorm Psychol 2001;110:363-371.

18. Blanchard JJ, Bellack AS, Mueser KT. Affective and social behavioral correlates of physical and social anhedonia in schizophrenia. J Abnorm Psychol 1994;103:719-728.

19. Katsanis J, Iacono WG, Beiser M, Lacey L. Clinical correlates of anhedonia and perceptual aberration in first-episode patients with schizophrenia and affective disorder. J Abnorm Psychol 1992;101:184-191.

20. Horan WP, Green MF, Kring AM, Nuechterlein KH. Does anhedonia in schizophrenia reflect faulty memory for subjectively experienced emotions? J Abnorm Psychol 2006;115:496-508.

21. Kentros MK, Terkelsen K, Hull J, Smith TE, Goodman M. The relationship between personality and quality of life in persons with schizoaffective disorder and schizophrenia. Qual Life Res 1997;6:118-122.

22. Hansson L. Determinants of quality of life in people with severe mental illness. Acta Psychiatr Scand Suppl 2006;(429):46-50.

23. Lysaker PH, Bell MD, Kaplan E, Bryson G. Personality and psychoso- 
cial dysfunction in schizophrenia: the association of extraversion and neuroticism to deficits in work performance. Psychiatry Res 1998;80: 61-68.

24. Blanchard JJ, Mueser KT, Bellack AS. Anhedonia, positive and negative affect, and social functioning in schizophrenia. Schizophr Bull 1998;24:413-424.

25. Schuck J, Leventhal D, Rothstein H, Irizarry V. Physical anhedonia and schizophrenia. J Abnorm Psychol 1984;93:342-344.

26. Herbener ES, Song W, Khine TT, Sweeney JA. What aspects of emotional functioning are impaired in schizophrenia? Schizophr Res 2008; 98:239-246.

27. Blanchard JJ, Panzarella C. Affect and Social Functioning in Schizophrenia. In: Mueser KT, Tarrier N, Editors. Handbook of Social Functioning in Schizophrenia. Needham Heights, MA: Allyn \& Bacon, Inc., 1998, p.181-196.

28. Velthorst E, Nieman DH, Becker HE, van de Fliert R, Dingemans PM, Klaassen R, et al. Baseline differences in clinical symptomatology between ultra high risk subjects with and without a transition to psychosis. Schizophr Res 2009;109:60-65.

29. Schlosser DA, Fisher M, Gard D, Fulford D, Loewy RL, Vinogradov S. Motivational deficits in individuals at-risk for psychosis and across the course of schizophrenia. Schizophr Res 2014;158:52-57.

30. Cooper S, Kring AM, Ellman LM. Attenuated positive psychotic symptoms and the experience of anhedonia. Early Interv Psychiatry 2017 [Epub ahead of print].

31. McGlashan TH, Miller TJ, Woods SW, Rosen JL, Hoffman RE, Davidson L. Structured Interview for Prodromal Syndromes (SIPS). Version 4.0. New Heaven: Yale University; 2003.

32. First MB, Gibbon M, Spitzer RL, Williams JBW. Structured Clinical Interview for DSM-IV Axis I Disorders: Non-Patients Edition (SCIDI/PS), Version 2. New York: New York State Psychiatric Institute Biometric Research; 1996.

33. First MB, Spitzer RL, Gibbon M, Williams JBW. Structured Clinical Interview for DSM-IV Axis I Disorders: Patient Edition (SCID-I/P), Version 2. New York: New York State Psychiatric Institute Biometric Research; 1996.

34. Andreasen NC. The Scale for the Assessment of Negative Symptoms (SANS). Iowa City: University of Iowa; 1983.

35. Lee HS. Korean Version Eysenck Personality Questionnaire. Seoul: Hakjisa; 1997.

36. Lee JS, Park HJ, Chun JW, Seok JH, Park IH, Park B, et al. Neuroanatomical correlates of trait anhedonia in patients with schizophrenia: A voxel-based morphometric study. Neurosci Lett 2011;489:110-114.

37. Lee JS, Kim ES, Kim EJ, Kim J, Kim E, Lee SK, et al. The relationship between self-referential processing-related brain activity and anhedonia in patients with schizophrenia. Psychiatry Res Neuroimaging 2016;
254:112-118.

38. Choi SH, Park IH, Ku J, Choi KM, Park M, Kim JJ. Comparison of the Characteristics of Anhedonia between Patients with Schizophrenia and Depressive Disorder. J Korean Neuropsychiatr Assoc 2010;49:570-577.

39. Lee CH, Kim JH. Relations of perceived stress, cognitive set, and coping behaviors to depression: a focus on freshmen's stress experiences. Korean J Counsel Psychother 1988;1:25-45.

40. Kim YC. Likableness and imagery ratings of 400 personality-describing adjectives. J Soc Sci 1984;2:17-32.

41. Bang M, Kim KR, Song YY, Baek S, An SK. Neurocognitive impairments in individuals at ultra-high risk for psychosis: Who will really convert? Aust N Z J Psychiatry 2015;49:462-470.

42. Andreasen NC. The Scale for the Assessment of Positive Symptoms (SAPS). Iowa City: University of Iowa; 1984.

43. Cornblatt BA, Auther AM, Niendam T, Smith CW, Zinberg J, Bearden $\mathrm{CE}$, et al. Preliminary findings for two new measures of social and role functioning in the prodromal phase of schizophrenia. Schizophr Bull 2007;33;688-702.

44. Lee SJ, Kim KR, Lee SY, An SK. Impaired social and role function in ultra-high risk for psychosis and first-episode schizophrenia: Its relations with negative sympotms. Psychiatry Investig 2017;14:186-192.

45. Holm S. A simple sequentially rejective multiple test procedure. Scand J Stat 1979;6:65-70.

46. Kroken RA, Johnsen E, Ruud T, Wentzel-Larsen T, Jørgensen HA. Treatment of schizophrenia with antipsychotics in Norwegian emergency wards, a cross-sectional national study. BMC Psychiatry 2009;9:24.

47. Jhung K, Park JY, Song YY, Kang JI, Lee E, An SK. Experiential pleasure deficits in the prodrome: a study of emotional experiences in individuals at ultra-high risk for psychosis and recent-onset schizophrenia. Compr Psychiatry 2016;68:209-216.

48. Cohen AS, Najolia GM, Brown LA, Minor KS. The state-trait disjunction of anhedonia in schizophrenia: potential affective, cognitive and social-based mechanisms. Clin Psychol Rev 2011;31:440-448.

49. Strauss GP, Ruiz I, Visser KH, Crespo LP, Dickinson EK. Diminished Hedonic response in neuroleptic-free youth at ultra high-risk for psychosis. Schizophr Res Cogn 2018;12:1-7.

50. Gruber J, Strauss GP, Dombrecht L, Mittal VA. Neuroleptic-free youth at ultrahigh risk for psychosis evidence diminished emotion reactivity that is predicted by depression and anxiety. Schizophr Res 2018;193: 428-434.

51. Song YY, Kang JI, Kim SJ, Lee MK, Lee E, An SK. Temperament and character in individuals at ultra-high risk for psychosis and with firstepisode schizophrenia: associations with psychopathology, psychosocial functioning, and aspects of psychological health. Compr Psychiatry 2013;54:1161-1168. 